\title{
Stakeholder engagement analysis - a bioethics dilemma in patient-targeted intervention: patients with temporomandibular joint disorders
}

\author{
Andre Barkhordarian, Gary Demerjian, Allison Jan, Nateli Sama, Mia Nguyen, Angela Du and Francesco Chiappelli*
}

\begin{abstract}
Modern health care in the field of Medicine, Dentistry and Nursing is grounded in fundamental philosophy and epistemology of translational science. Recently in the U.S major national initiatives have been implemented in the hope of closing the gaps that sometimes exist between the two fundamental components of translational science, the translational research and translational effectiveness. Subsequent to these initiatives, many improvements have been made; however, important bioethical issues and limitations do still exist that need to be addressed. One such issue is the stakeholder engagement and its assessment and validation. Federal, state and local organizations such as PCORI and AHRQ concur that the key to a better understanding of the relationship between translational research and translational effectiveness is the assessment of the extent to which stakeholders are actively engaged in the translational process of healthcare. The stakeholder engagement analysis identifies who the stakeholders are, maps their contribution and involvement, evaluates their priorities and opinions, and accesses their current knowledge base. This analysis however requires conceptualization and validation from the bioethics standpoint. Here, we examine the bioethical dilemma of stakeholder engagement analysis in the context of the person-environment fit (PE-fit) theoretical model. This model is an approach to quantifying stakeholder engagement analysis for the design of patient-targeted interventions. In our previous studies of Alzheimer patients, we have developed, validated and used a simple instrument based on the PE-fit model that can be adapted and utilized in a much less studied pathology as a clinical model that has a wide range of symptoms and manifestations, the temporomandibular joint disorders (TMD). The temporomandibular joint (TMJ) is the jaw joint endowed with sensory and motor innervations that project from within the central nervous system and its dysfunction can be manifested systemically in forms of movement disorders, and related pathological symptomatologies.

Currently, there is limited reliable evidence available to fully understand the complexity of the various domains of translational effectiveness, particularly in the context of stakeholder engagement and its assessment, validation as well as the bioethical implications as they pertain to evidence-based, effectivness-focused and patient-centered care.
\end{abstract}

Keywords: Bioethics, Translational science, Temporomandibular joint disorders, Patient-centered outcomes research, Stakeholders engagement analysis, Patient-evidence-provider fit, Patient-targeted intervention

\footnotetext{
* Correspondence: fchiappelli@dentistry.ucla.edu

Division of Oral Biology \& Medicine, UCLA School of Dentistry, 10833 Le

Conte Avenue, CHS 63-090 Los Angeles, CA, USA
} 


\section{Background}

The Patient Protection and Affordable Care Act (PPACA2010) [1] seeks to improve healthcare in the U.S., but it contains many controversial flaws and limitations. The pursuit of translational medicine, dentistry and nursing is based on the fundamentals of translational science, which is composed of two domains, the translational research and the translational effectiveness. Translational research refers to the scientific research the "bench-to-bedside" enterprise of translating knowledge from the basic sciences into the development of new treatments. It is the movement between basic research and patient-oriented research that leads to new or improved scientific understanding. Translational effectiveness refers to translating the findings and results into everyday practice. It facilitates the movement between patient-oriented research and populationbased research that leads to better patient outcomes, the implementation of best practices, and improved health status for patients and communities. Together they adhere to the hypothesis-driven scientific process that involves an intimate transaction of the systematic process of patientcentered outcomes research on the molecular and systems pathobiology of the patient, as well as the research synthesis process for the consensus of the best available evidence in patient-targeted interventions. Translational science as a whole aims to increase health literacy of the patients, caregivers and other stakeholders, which in turn demands significant improvements in evidence dissemination empowering them to actively participate in the decision-making process. However, a clear, well defined and bioethically sound procedural methodologies are still missing for assessing, evaluating and analyzing stakeholder engagement. Here, we examine this question in patients with Temporomandibular Joint Disorders (TMD) as a clinical model, because, while this pathology commences as a problem related to the field of dentistry, it can lead to systemic manifestations that involve medicine and nursing. We propose that stakeholder engagement analysis in translational science is bioethically sound, so long as it contributes to the psycho-cognitive, psychoemotional accommodation and well being of the stakeholder in the reality of the clinical condition at hand [2].

\section{The temporomandibular joint}

The temporomandibular joints are ginglymo-arthrodial bilateral articulations of the mandible with the maxillary bone of the frontal aspect of the skull, with a hinge-type ginglymal and a sliding arthrodial component. Encapsulated by a fibrous tissue, each joint consists of the condylar process of the mandible below, and the glenoid fossa of the temporal bone above. Between these bone surfaces lies the articular meniscal disc, held by tight fibers from below and looser fibers from the temporal bone superiorly, which creates an upper and a lower capsular space. A synovial membrane lines the inner facet of the capsules, and secretes the temporomandibular synovium that fills and lubricates the upper and lower spaces [3]. When adequate support by the relative occlusional position of the upper and lower teeth, the molars in particular, is lacking, the joint is progressively and chronically altered anatomically due to deregulated functions of masticatory muscles, which contributes to temporomandibular joint pathology [3].

The trigeminal nerve, the largest of the twelve cranial nerves is responsible for sensation in the head and neck. Of its three branches, the mandibular branch innervates the lower face and the temporomandibular joint. It projects somatic afferent fibers, and motor innervation to the masticatory musculature (i.e., special visceral efferent fibers) [3]. The trigeminal branches originate from the trigeminal ganglion, whence a single large sensory root enters the brainstem at the level of pons. A number of cranial nerves converge to the pons, besides the midpontine sensory and motor trigeminal projections. These include the abducens nerve (cranial nerve VI), which controls the lateral rectus muscle for movement of the eye, the facial nerve (VII), which controls the muscles of facial expression, and the vestibular-cochlear nerve (VIII), which transmits sound and equilibrium (balance) information from the inner ear to the brain. Together they impart to the pons its sensory regulation of hearing, equilibrium and movement, taste, and facial sensation to touch and pain, as well as its motor regulatory function for eye movement, facial expressions, chewing, swallowing, saliva and tear secretion, as well as movement coordination $[3,4]$.

Disorders of the temporomandibular joint encompass a spectrum of diverse conditions, and the term TMD describes a group of conditions with similar signs and symptoms that affect the termporomandibular joints, the muscles of mastication, or both. TMD is often characterized by aching of the muscles of mastication, pain and clicking or popping sounds upon opening and closing the jaw, and severely restricted jaw movement. In our previous studies, we showed that TMD contributes to the development and exacerbation of cervical dystonia and other movement disorders, most likely via neuritis of the auriculotemporal branch of the trigeminal nerve that feeds into the pontine region and controls head and body posture [5]. In those studies we also proposed that the proteomic signature of biomarkers in local (e.g., joint synovial fluid) and distal body fluids (e.g., saliva, cerebrospinal fluid) in a translational research modality would behoove patienttargeted TMD diagnosis and prognosis [6,7]. Our data obtained within the EBD-PBRN also indicated remarkable elevations in certain neuroimmune factors in the synovial fluid of TMD patients that were significantly and positively correlated with diagnostic imaging-rated severity of the TMD condition [8] (Barkhordarian et al., under 
review) confirming the intimate coexistence of neuroimmunological and neuropathology complexities with TMD.

\section{Stakeholders in translational medicine}

Translational medicine rests on the fundamental principles of stakeholder engagement [2]. Stakeholders are important partners in the clinical decision-making process $[2,9,10]$, particularly in the context of patient-centered healthcare home/neighborhoods for optimizing patienttargeted interventions. However, the evidence on the effectiveness and the bioethics of stakeholder engagement still remains to be limited [11,12].

Stakeholder theory originally defined stakeholders as those individuals without whose support and feedback an organization, or a project within an organization cannot subsist [13]. In the context of translational medicine, stakeholders are: basic scientists, clinicians/clinical researchers, epidemiologists, health services researchers, patients, family members, caregivers, patient advocates, social workers, insurance carriers, legal advisers. They provide multidimensional contributions to patient-targeted endeavors in evidence-based, effectiveness-focused clinical decision-making processes $[8,14]$. As shown in Figure 1 stakeholders in modern healthcare engage in translational medicine as:

1. Primary stakeholders: those individuals ultimately and directly affected, either positively or negatively, by the healthcare outcomes (e.g., patients, the immediate family members, caregivers of patients who cannot represent themselves),

2. Key stakeholders: individuals who may or may not be primary stakeholders, but have a significant influence on the decision-making process (e.g., relatives, friends or caregivers empowered by a legal document or directive to make healthcare decisions on behalf of the patient),

3. Secondary stakeholders: individuals indirectly affected by the outcomes, or indirectly involved in the patient's care process,

4. Allied stakeholders: individuals who are involved in the patient's care, but are indirectly affected by the healthcare outcome (e.g., medical, dental, nursing and pharmacy staff, other hospital employees, insurance agents, legal staff and lawyers).

The critical questions and issues of bioethics in translational medicine has remained to be addressed in part because individuals who judge themselves to be stakeholders engage de facto (in practice but not necessarily ordained by law) as stakeholders raising issues of liability and protection of the confidentiality and security of health care information. Incontrovertible evidence establishes that stakeholder engagement is rarely uniform: not all stakeholders have the same roles and degree of involvement in the healthcare process. Therefore, assessing stakeholder engagement (Table 1) while necessary and critical for translational medicine may be bioethically

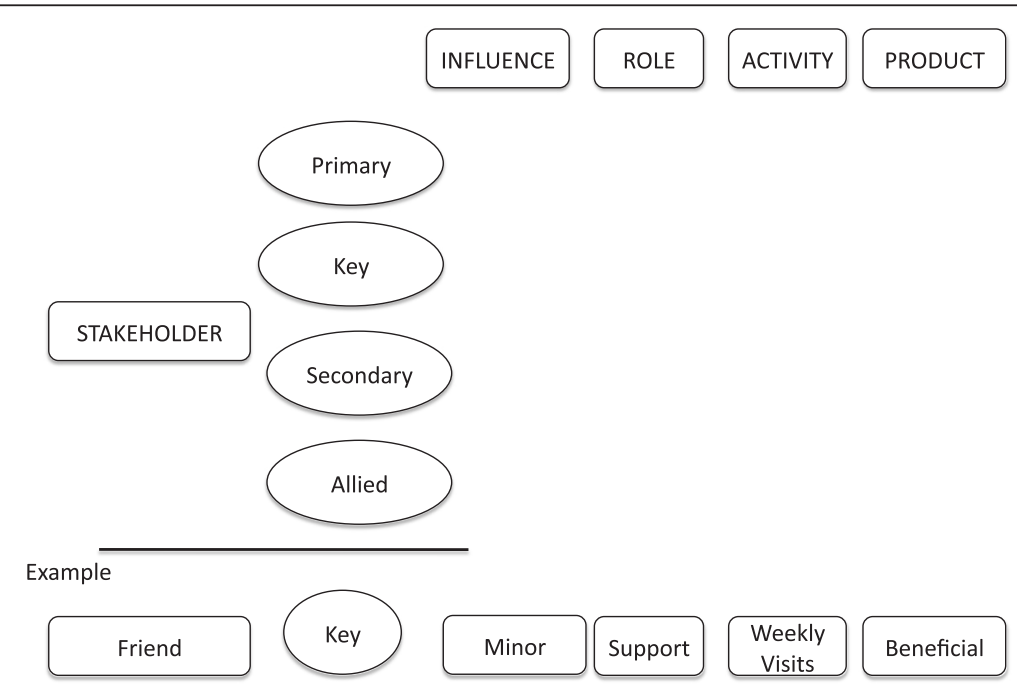

Figure 1 Stakeholder matrix. The figure shows a simplified stakeholder matrix to exemplify the process of stakeholder "mapping". As shown in the example provided in the figure, each stakeholder is identified as primary, key, secondary or allied. The stakeholder's influence, role activity, and other characteristics are tabulated in the matrix, as well as the stakeholder's effect and outcome of the healthcare intervention process. In the example provided, we have a "friend", who could be a secondary stakeholder identified in this case as a key stakeholder, presumably because of possessing a Power of Attorney or directive who makes healthcare decisions on behalf of the patient. We observe the influence of this friend to be, at this present moment, relatively minor as visits are relatively rare. Yet, we recognize beneficial outcomes from those visits (it could also be the case that visits have a seriously detrimental outcome on the patient). This tabulation is prepared for every stakeholder, and is regularly revisited and updated. 
Table 1 The seven principal steps of stakeholder analysis

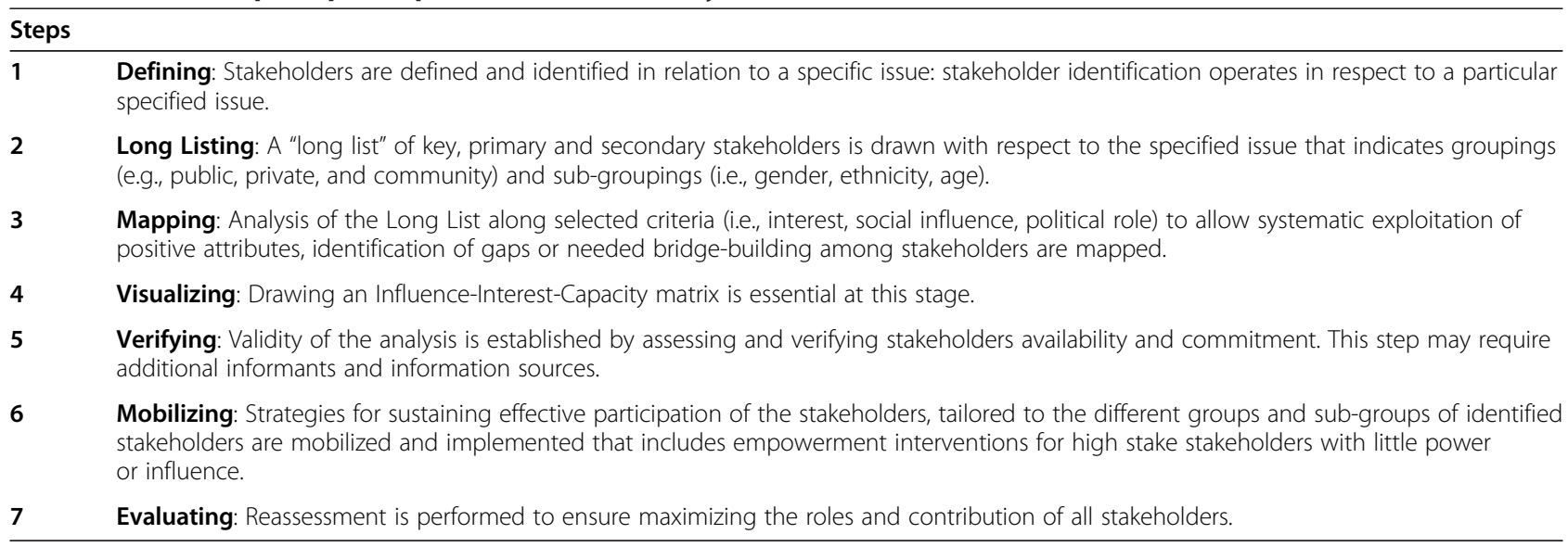

restricted, biased and compromised. There is a need for concerted research efforts to be directed towards the development and validation of novel analytical tools to establish the nature, level (or quantity), and quality of stakeholder participation in healthcare [15].

Stakeholder analysis must evolve as systematic, sequential scientific process, subject to a stringent process of construct validation designed to identify and characterize sub-constructs related to stakeholder engagement. Stakeholders' roles, involvement and function must be quantified, including their (1) actions and position; (2) ability and intent to influence implementation; (3) motivation to participate; and (4) capability to change and adapt as the care situation evolves, and calls for adaptive management [2,16-18]. Reliable tools must be developed, as mentioned, to that end, which must be bioethically coherent and contemporaneously validated to quantify stakeholders' beneficial input.

In order to assess the reliability of these novel instruments, the analyses of inter-rater reliability and coefficient of agreement [2], as well as generalizability (G) theory validation will be required. The generalizability validation process ensures a flexible approach to simultaneously estimate multiple sources of measurement error variance (i.e., facets), while permitting to generalize the findings of the main analysis across the different study facets. G theory leads to re-calculate the reliability and minimal detectable changes across a variety of combination conditions of these facets. In brief, the G mode of validation of stakeholder engagement analysis will engender the selection of optimal settings minimizing the number of required measures $[2,19]$.

In our preliminary studies (data not included) we have examined the criteria and processes necessary to improve stakeholder engagement by raising their health literacy. We were able to demonstrate that bioethically sound training in communication skills could significantly improve physicians-patient relationship, reduce inappropriate use of medical information, and raise stakeholder engagement [20].

\section{The Person-Environment (PE) fit theoretical model}

To best examine the role and fit of stakeholders in the context of patient-targeted interventions in translational medicine, we considered the well-established and validated theoretical PE fit model. PE-fit establishes the degree to which a person is compatible with the environment, and determines the extent to which someone's personality and set of skills are adaptable with the environmental demands. It defines the degree to which an individual can successfully adjust to the imperatives of the surrounding. The model arose from the Personality-Job Fit theory, which states in brief that a person's personality, training and skills determine one's adaptability and synergy in an organization and a work environment. A perceived (i.e., subjective) or real (i.e., objective) misfit between the person and environment leads to impaired outcomes on specific tasks, job performance, satisfaction and quality of professional and personal life. From an analytical standpoint, the model offers a framework for establishing and predicting how the person's real and perceived characteristics and skills will fit and adapt to the real and perceived requisites and demands of the work environment. Because the perception of fit of an individual within a given environment is dependent upon one's ability to discern between perception and reality, the model reveals preventive intervention toward improving the person's well-being, should it be sub-optimal due to constraining factors in the environment [2,21-23].

The model evolved to address outcomes of ill-health, consequential to the psycho-emotional stress associated with a perceived or real lack of fit, between an individual and the environment. Further evolution rendered the model more widely applicable across a variety of settings in translational medicine, including our study of evidence- 
based health care for caregivers (i.e., primary stakeholders) of patients with Alzheimer's disease [24,25].

In that work, we constructed and validated a simple instrument (in-house questionnaire), based on the same criteria described in the literature [21], which consisted of 15 components aimed at quantifying the subjective and objective perceptions of the caregivers and of the immediate demands of the caregiving responsibilities (Table 2) [25]. The data were analyzed along four simple criteria:

- The principal subjective caregiver-environment fit: fit between the subjective perception of the caregiver and his/her perceived responsibilities;

- The secondary objective caregiver-environment fit: fit between the objective abilities of caregiver in the face of the objective demands of the caregiving responsibilities;

- The reality contact: correspondence between the subjective perception of the responsibilities of caregiving and the objective reality posed by these responsibilities; and

- The accuracy, or accessibility, of the self: correspondence between the subjective views and objective reality of the caregiver' abilities to face these demanding responsibilities.

The subjective person-environment fit $(\mathrm{Fs})$ is the result of the interdependent relationship between the person's subjective assessment of self (Ps) and his or her subjective evaluation of the environment (Es). In a parallel fashion, the objective environment (Eo) and the objectively assessed person's abilities to meet its demands ( $\mathrm{Po}$ ) yield a quantification of the objective person-environment fit (Fo). These relationships can be summarized quantitatively as Fo $=$ Eo-Po and Fs = Es-Ps, a can reflect the demand, or need on the part of the person $(\mathrm{Np})$, or the environment $(\mathrm{Ne})$ to actualize fit; and the given abilities of the person (Gp), or the given attributes of the environment (Ge) that facilitates fit. The objective person-environment fit (Fo) is a complex function of the difference (delta, $\Delta$ ) between the attributes of the environment $(\mathrm{Ge})$ and the need on the part of the person $(\mathrm{Np})$ or the environment $(\mathrm{Ne})$ to actualize fit. In the same vein, subjective personenvironment fit $(\mathrm{Fs})$ is a function of the delta between the attributes of the person (Gp) and the need of the environment $(\mathrm{Ne})$ to facilitate fit.

The validity and the reliability of the instrument were tested in 200 subjects stratified, based on clinical exam among the groups of senile dementia of the Alzheimer's type of stages 1-5 on the Global Assessment Scale (age range: 55-70), and of age-matched non-Alzheimer's type dementias that included vascular dementias, Parkinson's dementia, and dementia with Lewy bodies. We also included control subjects with no signs of dementia and of the same age range. Taken together, the inferences derived from this simple instrument provided a critical element to ensure optimal utilization of caregivers in the patient-targeted evidence-based clinical intervention for patients with Alzheimer's disease [25].

Currently we are in the process of developing a new instrument (questioner) in order to incorporate bioethical issues in the assessment of the stakeholder engagement for patients with TMD. Ongoing studies are validating a revision and expansion of the original instrument, based in part on the ethical dialectics proposed in the literature and above [26]. Some of the bioethical issues that need to be incorporated in the new instrument are highlighted (Table 3).

Table 2 PE fit for caregivers of patients with Alzheimer's disease [25]

\begin{tabular}{lll}
\hline 1 & Overall perception of health & Subjective assessment by the caregiver, overall Fit \\
3 & Perceived energy level & Subjective, caregiver (Person) \\
4 & Perceived mood of patient & Subjective, caregiving responsibilities (Environment) \\
5 & Perceived lifestyle of patient & Subjective, caregiving responsibilities (Environment) \\
6 & Perceived memory of patient & Subjective, caregiving responsibilities (Environment) \\
7 & Perceived family relationships & Subjective, caregiving responsibilities (Environment) \\
8 & Perceived relationship with spouse & Subjective, caregiving responsibilities (Environment) \\
9 & Perceived relationship with friends & Subjective, caregiving responsibilities (Environment) \\
10 & Perceived sense of self & Subjective, caregiver (Person) \\
11 & Ability to perform household chores & Objective, caregiving responsibilities (Environment) \\
12 & Enjoyment of leisure & Objective, caregiving responsibilities (Environment) \\
13 & Ability to hold financial responsibilities & Subjective, caregiver (Person) \\
14 & Perception that own life is ending & Objective, caregiver (Person) \\
15 & Overall life satisfaction & Objective, caregiver (Person) \\
\hline
\end{tabular}


Table 3 Stakeholder engagement fit general model

\begin{tabular}{lll}
\hline & Subjective & Objective \\
\hline Stakeholder & $\begin{array}{l}\text { Perception of the stakeholder's energy level, mood, } \\
\text { abilities and skills, and willingness to meet the } \\
\text { demands imposed by their engagement in the } \\
\text { clinical decision-making process for patient-targeted } \\
\text { intervention. }\end{array}$ & $\begin{array}{l}\text { Effectiveness in taking on own role as stakeholder, facing } \\
\text { financial hardship, adapting in changing life's role and } \\
\text { routine, and controlling psycho-emotional strain and stress } \\
\text { associated with the demands imposed by their engagement } \\
\text { in patient-targeted intervention. }\end{array}$ \\
$\begin{array}{ll}\text { Engagement in the clinical } \\
\text { decision-making process }\end{array}$ & $\begin{array}{l}\text { Stakeholder's understanding and knowledge of the } \\
\text { clinical condition at hand, of the options for treatment, } \\
\text { of the best available evidence (i.e., health literacy). }\end{array}$ & $\begin{array}{l}\text { Bioethical concerns, including privacy, bias, ensuring } \\
\text { incremental risks" [28], and values [29]. }\end{array}$ \\
\hline
\end{tabular}

To further establish the applicability of the PE fit approach to bioethical concerns in translational medicine, we propose the person-environment fit model to a patient-evidence-provider best-fit model that is grounded on the same principles outlined above [2]. In this more complex approach, eight primary outcomes were identified (below) to describe the relation among objective and subjective patients, evidence and providers. These domains are used in order to develop specific criteria for the stakeholder engagement assessment tool.

1. Patient objective - Evidence objective - Provider Objective: optimal translational medicine

2. Patient objective - Evidence objective - Provider Subjective: sub-optimal translational medicine, with potential bias on the part of the provider

3. Patient objective - Evidence subjective - Provider subjective: health care based on the evidence, with added concern of potentially biased provider

4. Patient Objective - Evidence subjective - Provider objective: health care based on the evidence, provider unbiased

5. Patient subjective - Evidence Objective - Provider subjective: although the best available evidence is used, caveat of bias due subjective patient and provider

6. Patient subjective - Evidence subjective - Provider subjective: evidence-based of the evidence, with all possible drawbacks of subjective assessments

7. Patient subjective - Evidence objective - Provider objective: attempt is made for translational medicine, but patient is biased with pre-conceived notions: provider must help patient "un"learn the misguided information before the best available evidence can be given

8. Patient subjective - Evidence subjective - Provider objective: provider needs to be taught how to gain access to the best available evidence in order to carry out translational medicine effectively [2].

\section{Conclusion}

Primary areas of bioethical concern subsist in the context of patient-targeted intervention in translational medicine. They are: (1) selection bias in the sampling process, (2) invasiveness of the screening and treatment protocols, (3) infraction to the fundamental principles of human rights and human dignity, and (4) reliable process of research synthesis for obtaining the best available evidence for patient-targeted intervention, and unbiased dissemination and utilization in specific clinical settings. The latter pertains to the domain of translational effectiveness, which we have discussed previously [30], and involves the issues of stakeholder engagement assessment and analysis we have considered here, in line, with the need for "individualized equal care" proposed by others [27]. Incontestable compelling evidence shows that from a bioethical standpoint, patients and their representatives have the right to be included in decisions that pertain to patienttargeted interventions. It is also self-evident that this privilege cannot be extended to all stakeholders, and that stakeholder engagement analysis is needed to contribute positive impact on the transparency and accountability of translational science. Therefore, reaching a shared understanding of ethical roles and responsibilities amongst stakeholders require dialogue and reciprocal awareness of limitations.

\section{Abbreviations \\ AHRQ: Agency for Healthcare Research Quality; EBD-PBRN: Evidence-Based Decisions Practice-Based Research Network; PE fit: Person-Environment fit; PPACA: Patient Protection and Affordable Care Act; PCORI: Patient Centered Outcomes Research Institute; $\mathrm{NIH}$ : National Institutes of Health; \\ TMD: Temporomandibular joint disorders.}

\section{Competing interests}

The authors declare that they have no competing interest.

\section{Authors' contributions}

$A B$ : research lead of the TMD project. GD: clinical lead of the TMD project. AJ: contributed to the conceptualization of fundamental bioethics in translational science. NS: contributed to the conceptualization of the PEP Fit model. MN \& $\mathrm{AD}$ : contributed to the psychoneuroimmunology. FC: senior lead of the TMD project. All authors read and approved the final manuscript.

\section{Authors' information}

AB: Project Scientist, Div. Oral Biology \& Medicine, Dentistry, UCLA osteoimmunology specialist.

GD: Project Scientist, Div. Oral Biology \& Medicine, Dentistry, UCLA - Private Practice: TMJ Specialist.

AJ: UCLA undergraduate student - pre-dental student.

NS: UCLA undergraduate student - pre-dental student.

MN: UCLA undergraduate student - pre-dental student.

AD: UCLA undergraduate student - pre-dental student.

FC: Professor, UCLA Dentistry - translational science specialist \&

psychoneuroenocrine-immunology specialist. 


\section{Acknowledgments}

The authors thank the Evidence-Based Decisions Active Groups of Stakeholders (EBD-AGS) of the EBD-Practice-Based Research Network (ebd-pbrn.org), and the students and colleagues of the EBD Study Group. Funded in part by UCLA Senate grant and Fulbright Specialist grant (5077) to FC.

Received: 19 September 2014 Accepted: 16 December 2014

Published online: 20 January 2015

\section{References}

1. US Government Publishing Office. Public Law 111-148-The Patient Protection and Affordable Care Act. 2010. http://www.gpo.gov/fdsys/pkg/PLAW-111publ 148/pdf/PLAW-111publ148.pdf. Accessed 14 Oct 2014.

2. Chiappelli F. Fundamentals of Evidence-Based Health Care and Translational Science. Heidelberg, DE: Springer-Verlag; 2014.

3. Alomar X, Medrano J, Cabratosa J, Clavero JA, Lorente M, Serra I, et al. Anatomy of the temporomandibular joint. Semin Ultrasound CT MR. 2007:28:170-83.

4. Pritchard TE, Alloway D. Medical neuroscience. Raleigh, NC: Hayes Barton Press; 1999.

5. Sims AB, Stack BC, Demerjian GG. Spasmodic torticollis: the dental connection. Cranio. 2012;30:188-93.

6. Barkhordarian A, Ajaj R, Ramchandani MH, Demerjian G, Cayabyab R, Danaie S, Ghodousi N, Iyer N, Mahanian N, Phi L, Giroux A, Manfrini E, Neagos N, Siddiqui M, Cajulis OS, Brant X, Shapshak P, Chiappelli F: Osteoimmunopathology in HIV/AIDS. A Translational Evidence-Based Perspective. Pathology Research International, 2011, Article ID 359242 epub 21 May 2011.

7. Demerjian $\mathrm{GG}$, Sims $A B$, Stack BC. Proteomic signature of Temporomandibular Joint Disorders (TMD): Toward diagnostically predictive biomarkers. Bioinformation. 2011:5:282-4.

8. Barkhordarian A, Demerjian G, Chiappelli F. Systemic correlates and local responses to Temporomandibular Joint Disorders. 94th AAAS-PD Annual Meeting, Las Vegas, NV; 15-19 June 2013.

9. Wu RC, Tran K, Lo V, O'Leary KJ, Morra D, Quan SD, et al. Effects of clinical communication interventions in hospitals: a systematic review of information and communication technology adoptions for improved communication between clinicians. Int J Med Inform. 2012;81:723-32.

10. Goodman SN, Gerson J. Mechanistic Evidence in Evidence-Based Medicine: A Conceptual Framework [Internet]. Rockville (MD): Agency for Healthcare Research and Quality (US); 2013. Jun. AHRQ Methods for Effective Health Care Report No: 13-EHC042-EF.

11. Chiappelli F. The Patient-Centered Dental Home Model. Dental Hypotheses. 2011:2:105-12.

12. Arend J, Tsang-Quinn J, Levine C, Thomas D. The patient-centered medical home: history, components, and review of the evidence. Mt Sinai J Med. 2012;79:433-50.

13. Freeman R, Edward R, Reed DL. Stockholders and Stakeholders: A new perspective on Corporate Governance. Calif Manag Rev. 1983;25:88-106.

14. Qian M, Wu D, Wang E, Marincola FM, Wang W, Rhodes W, et al, Development and promotion in translational medicine: Perspectives from 2012 sino-american symposium on clinical and translational medicine. Clin Trans Med. 2010;1:25-30

15. Deverka PA, Lavallee DC, Desai PJ, Esmail LC, Ramsey SD, Veenstra DL, et al. Stakeholder participation in comparative effectiveness research: defining a framework for effective engagement. J Comparative Effect Res. 2012;1:181-94

16. de Almeida Moura J, Carvalho Costa B, de Faria RMD, Figueiredo Soares T, Perlatto Moura E, Chiappelli F. Improving communication skill training in patient centered medical practice for enhancing rational use of laboratory tests: The core of bioinformation for leveraging stakeholder engagement in regulatory science. Bioinformation. 2013:9:718-20.

17. Fletcher A, Guthrie J, Steane P, Roos G, Pike S. Mapping stakeholder perceptions for a third sector organization. J Intellect Cap. 2003:4:505-27.

18. Aarons GA, Wells RS, Zagursky K, Fettes DL, Palinkas LA. Implementing Evidence-Based Practice in Community Mental Health Agencies: A Multiple Stakeholder Analysis. Am J Public Health. 2009:99:2087-95.

19. Strauss ME, Smith GT. Construct Validity: Advances in Theory and Methodology. Annu Rev Clin Psychol. 2009;5:1-25.

20. Shavelson RJ, Webb NM. Generalizability Theory: 1973-1980. Br J Math Stat Psychol. 1981:34:133-66.

21. French Jr JRP, Rodgers WL, Cobb S. Adjustment as person-environment fit. In: Coelho G, Hamburg D, Adams J, editors. Coping and adaptation. New York: Basic Books; 1974. p. 316-33.
22. Caplan RD, Harrison RV. Person-environment fit theory: Some history, recent developments, and future directions. J Soc Issues. 1993:49:253-75.

23. Edwards JR, Caplan RD, Harrison RV. Person-environment fit theory: Conceptual foundations, empirical evidence, and directions for future research. In: Cooper $\mathrm{CL}$, editor. Theories of organizational stress. Oxford, GB: Oxford University Press; 1998. p. 28-67.

24. Edwards JR, Cooper CL. The person-environment fit approach to stress: Recurring problems and some suggested solutions. J Organ Behav. 1990;11:293-307.

25. Chiappelli F, Manfrini E, Edgerton M, Rosenblum M, Kristine D, Cajulis KD, et al. Clinical Evidence and Evidence-Based Dental Treatment of Special Populations: Patients with Alzheimer's Disease. California Dental Assoc J. 2006;34:439-47.

26. Loughlin M, Bluhm R, Drozdstoj S, Stoyanov DS, Buetow S, Upshur REG, et al. Explanation, understanding, objectivity and experience. J Eval Clin Pract. 2013;19:415-21.

27. Maglo KN. Group-Based and Personalized Care in an Age of Genomic and Evidence-Based Medicine: A Reappraisal. Perspect Biol Med. 2012;55:137-54.

28. Petrini C. Ethical Issues in Translational Research. Perspect Biol Med. 2010:53:517-33.

29. de Melo-Martín I, Intemann K. Interpreting Evidence: Why Values Can Matter As Much As Science. Perspect Biol Med. 2012:55:59-70.

30. Barkhordarian A, Ramchandani MH, Dousti M, Kelly-Gleason L, Chiappelli F. Disseminating the best available evidence: New challenges in public reporting of health care. Bioinformation. 2012;8:293-5.

\section{Submit your next manuscript to BioMed Central and take full advantage of:}

- Convenient online submission

- Thorough peer review

- No space constraints or color figure charges

- Immediate publication on acceptance

- Inclusion in PubMed, CAS, Scopus and Google Scholar

- Research which is freely available for redistribution 